Article

\title{
Chaos Control and Synchronization of a Hyperchaotic Zhou System by Integral Sliding Mode control
}

\section{Yashar Toopchi * and Jidong Wang}

School of Electrical and Computer Engineering (SECE), University of RMIT, GPO Box 2476V, Melbourne, Victoria, 3001, Australia; E-Mail: jidong.wang@rmit.edu.au

* Author to whom correspondence should be addressed; E-Mail: s3467252@ student.rmit.edu.au; Tel.: +61-3-9925-5306.

External Editor: J. A. Tenreiro Machado

Received: 25 September 2014; in revised form: 25 November 2014 / Accepted: 3 December 2014 / Published: 12 December 2014

\begin{abstract}
In this paper, an adaptive integral sliding mode control scheme is proposed for synchronization of hyperchaotic Zhou systems. In the proposed scheme, an integral sliding mode control is designed to stabilize a hyperchaotic Zhou system with known parameters to its unstable equilibrium at the origin. The control is then applied to the synchronization of two identical systems, i.e., a slave and a master hyperchaotic Zhou system with unknown parameters. The adaptive control mechanism introduced synchronizes the systems by estimating the unknown parameters. Simulation results have shown that the proposed method has an excellent convergence from both speed and accuracy points of view, and it outperforms Vaidyanathan's scheme, which is a well-recognized scheme in this area.
\end{abstract}

Keywords: hyperchaotic Zhou system; adaptive controller and synchronization; integral sliding mode control; Lyapunov stability theory

\section{Introduction}

Hyperchaotic systems have been defined as chaotic systems having more than one positive Lyapunov exponent. Hyperchaotic systems exhibit complex dynamics and characteristics, such as high capacity, high security and high efficiency. Some classical hyperchaotic systems are the hyperchaotic Rössler system [1], hyperchaotic Lorenz-Haken system [2], hyperchaotic Chua's circuit [3], hyperchaotic Chen system [4], hyperchaotic Lü system [5], etc. The control of a chaotic system is to devise a state feedback 
control law to stabilize the system around its unstable equilibrium points [6,7]. Active control [8] is applied when the system parameters are known, and adaptive control [9] is applied when the system parameters are unknown. Synchronization of chaotic systems is said to occur when a chaotic attractor drives another chaotic attractor. In the last two decades, there has been considerable interest devoted to the synchronization of chaotic and hyperchaotic systems. In their seminal paper in 1990, Pecora and Carroll [10] introduced a method to synchronize two identical chaotic systems and showed that it was possible for some chaotic systems to be completely synchronized. Subsequently, chaos synchronization has been applied in a wide variety of fields, including physics [11], chemistry [12], ecology [13], secure communications [14,15], cardiology [16], robotics [17], complex dynamical networks [18], etc. Some common methods applied to the chaos synchronization problem are the active control method $[19,20]$, adaptive control method [21,22], sampled-data feedback method [23], time-delay feedback method [24], backstepping method [25,26], sliding mode control method [27-36], etc. In this paper, a new scheme based on the adaptive integral sliding mode control for the chaos synchronization of two identical hyperchaotic Zhou systems [37] is derived. The sliding mode control method is often used because of its inherent advantages of easy realization, fast response and good transient performance, as well as its insensitivity to parameter uncertainties and external disturbances. This paper has been organized as follows. In Section 2, an analysis and description of the hyperchaotic Zhou system is presented. In Section 3, the results of the proposed method for the adaptive control of the hyperchaotic Zhou system with unknown parameters, as well as results of the proposed method for the adaptive synchronization of the identical hyperchaotic Zhou systems with unknown parameters is provided. The corresponding simulation results are shown in Section 4. Finally, a discussion and the conclusion are given in Section 5.

\section{System Description}

The Zhou system [37] is described by the four-dimensional dynamics:

$$
\left\{\begin{array}{l}
\dot{x}_{1}=a\left(x_{2}-x_{1}\right)+x_{4} \\
\dot{x}_{2}=c x_{2}-x_{1} x_{3} \\
\dot{x}_{3}=-b x_{3}+x_{1} x_{2} \\
\dot{x}_{4}=d x_{1}+0.5 x_{2} x_{3}
\end{array}\right.
$$

where $x_{1}, x_{2}, x_{3}$ and $x_{4}$ are the states and $a, b, c$ and $d$ are the constant, positive parameters of the system.

The Zhou system exhibits hyperchaotic behavior when the parameter values are:

$$
a=35, \quad b=3, \quad c=12, \quad 0<d<34.5 .
$$

Figure 1 depicts the hyperchaotic phase portrait of the hyperchaotic Zhou system, where, for simulation, the values of $a, b$ and $c$ are as given in Equation (2) and the value of $d$ is chosen as $d=1$.

When the parameter values are taken as in Equation (2) for the Zhou system, the system linearization matrix at the equilibrium point $E_{0}=[0,0,0,0]$ is given by:

$$
A=\left[\begin{array}{cccc}
-a & a & 0 & 1 \\
0 & c & 0 & 0 \\
0 & 0 & -b & 0 \\
d & 0 & 0 & 0
\end{array}\right]
$$


Equation (4) contains the eigenvalues as:

$$
\lambda_{1}=c, \quad \lambda_{2}=-b, \quad \lambda_{3}=\frac{-a+\sqrt{a^{2}+4 a}}{2}, \quad \lambda_{4}=\frac{-a-\sqrt{a^{2}+4 a}}{2} .
$$

Since $\lambda_{1}$ is a positive eigenvalue of $A$, it is immediate from Lyapunov stability theory [38] that the hyperchaotic Zhou system Equation (1) is unstable at the equilibrium point $E_{0}=[0,0,0,0]$.

Figure 1. (a) Phase portrait of the hyperchaotic Zhou system; (b) time-history of states $x_{1}$, $x_{2}, x_{3}$ and $x_{4}$.
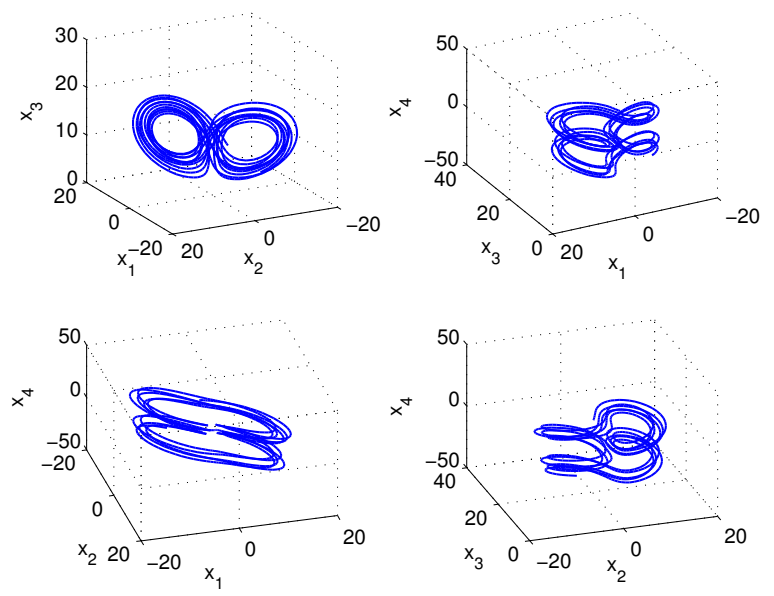

(a)

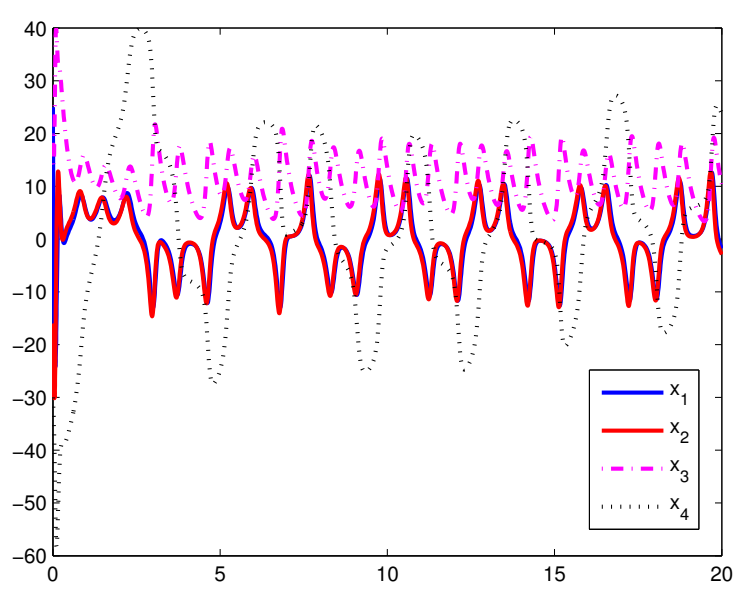

(b)

\section{Sliding Mode Control of Hyperchaotic Zhou System}

In this section, an integral sliding mode controller [38] for globally stabilizing the hyperchaotic Zhou system [37] with unknown parameters is designed. Thus, the controlled hyperchaotic Zhou system is described by:

$$
\left\{\begin{array}{l}
\dot{x}_{1}=a\left(x_{2}-x_{1}\right)+x_{4}+u_{1} \\
\dot{x}_{2}=c x_{2}-x_{1} x_{3}+u_{2} \\
\dot{x}_{3}=-b x_{3}+x_{1} x_{2}+u_{3} \\
\dot{x}_{4}=d x_{1}+0.5 x_{2} x_{3}+u_{4}
\end{array}\right.
$$

where $u_{1}, u_{2}, u_{3}$ and $u_{4}$ are feedback controllers to be designed based on the states $x_{1}, x_{2}, x_{3}$ and $x_{4}$ in a way that the controlled system Equation (3) globally converges to the origin asymptotically.

Based on the sliding mode theory [36,38], the integral sliding surface of each $x_{i}, i=1, \cdots, 4$ is defined as below:

$$
s_{i}=\left(\frac{d}{d t}+\lambda_{i}\right)\left(\int_{0}^{t} x_{i}(\tau) d \tau\right)=x_{i}+\lambda_{i} \int_{0}^{t} x_{i}(\tau) d \tau, \quad i=1, \ldots, 4
$$

The derivative of the equation Equation (6) yields:

$$
\dot{s}_{i}=\dot{x}_{i}+\lambda_{i} x_{i}, \quad i=1, \ldots, 4
$$


The Hurwitz condition is realized if $\lambda_{i}>0, i=1, \ldots, 4$.

Based on the exponential reaching law [38]:

$$
\dot{s}_{i}=-\eta_{i} \operatorname{sgn}\left(s_{i}\right)-k_{i} s_{i}, \quad i=1, \ldots, 4
$$

Comparing Equations (7) and (8) gives:

$$
\left\{\begin{array}{l}
\dot{x_{1}}+\lambda_{1} x_{1}=-\eta_{1} \operatorname{sgn}\left(s_{1}\right)-k_{1} s_{1} \\
\dot{x_{2}}+\lambda_{2} x_{2}=-\eta_{2} \operatorname{sgn}\left(s_{2}\right)-k_{2} s_{2} \\
\dot{x_{3}}+\lambda_{3} x_{3}=-\eta_{3} \operatorname{sgn}\left(s_{3}\right)-k_{3} s_{3} \\
\dot{x_{4}}+\lambda_{4} x_{4}=-\eta_{4} \operatorname{sgn}\left(s_{4}\right)-k_{4} s_{4}
\end{array}\right.
$$

Using the result in Equation (5), then Equation (8) is rewritten as:

$$
\left.\begin{array}{rl}
a\left(x_{2}-x_{1}\right)+x_{4}+u_{1}+\lambda_{1} x_{1} & =-\eta_{1} \operatorname{sgn}\left(s_{1}\right)-k_{1} s_{1} \\
c x_{2}-x_{1} x_{3}+u_{2}+\lambda_{2} x_{2} & =-\eta_{2} \operatorname{sgn}\left(s_{2}\right)-k_{2} s_{2} \\
-b x_{3}+x_{1} x_{2}+u_{3}+\lambda_{3} x_{3} & =-\eta_{3} \operatorname{sgn}\left(s_{3}\right)-k_{3} s_{3} \\
d x_{1}+0.5 x_{2} x_{3}+u_{4}+\lambda_{4} x_{4} & =-\eta_{4} \operatorname{sgn}\left(s_{4}\right)-k_{4} s_{4}
\end{array}\right\}
$$

Then, the control laws are obtained for $i=1, \ldots, 4$ as follows:

$$
\left\{\begin{array}{l}
u_{1}=-a\left(x_{2}-x_{1}\right)-x_{4}-\lambda_{1} x_{1}-\eta_{1} \operatorname{sgn}\left(s_{1}\right)-k_{1} s_{1} \\
u_{2}=-c x_{2}+x_{1} x_{3}-\lambda_{2} x_{2}-\eta_{2} \operatorname{sgn}\left(s_{2}\right)-k_{2} s_{2} \\
u_{3}=b x_{3}-x_{1} x_{2}-\lambda_{3} x_{3}-\eta_{3} \operatorname{sgn}\left(s_{3}\right)-k_{3} s_{3} \\
u_{4}=-d x_{1}-0.5 x_{2} x_{3}-\lambda_{4} x_{4}-\eta_{4} \operatorname{sgn}\left(s_{4}\right)-k_{4} s_{4}
\end{array}\right.
$$

Theorem 1. The hyperchaotic Zhou system in Equation (5) with the arbitrary initial condition $x(0) \in \mathbb{R}^{4}$ using the control law in Equation (11) and with $\lambda_{i}, \eta_{i}$ and $k_{i}>0$ is globally asymptotically stable.

Proof. Let us consider the following Lyapunov function:

$$
V=\frac{1}{2}\left(s_{1}^{2}+s_{2}^{2}+s_{3}^{2}+s_{4}^{2}\right)
$$

where $s_{i}, i=1, \ldots, 4$, the same as the ones in Equation (6), has been defined for the response of the Zhou system in Equation (5), where the system in Equation (5) utilizes command signals in Equation (11). The derivative of Equation (12) gives:

$$
\dot{V}=s_{1} \dot{s}_{1}+s_{2} \dot{s}_{2}+s_{3} \dot{s}_{3}+s_{4} \dot{s}_{4}
$$

By substituting Equation (8) into Equation (13):

$$
\begin{aligned}
\dot{V}= & s_{1}\left(-\eta_{1} \operatorname{sgn}\left(s_{1}\right)-k_{1} s_{1}\right)+s_{2}\left(-\eta_{2} \operatorname{sgn}\left(s_{2}\right)-k_{2} s_{2}\right)+s_{3}\left(-\eta_{3} \operatorname{sgn}\left(s_{3}\right)-k_{3} s_{3}\right) \\
& +s_{4}\left(-\eta_{4} \operatorname{sgn}\left(s_{4}\right)-k_{4} s_{4}\right) \\
= & -\eta_{1}\left|s_{1}\right|-k_{1} s_{1}^{2}-\eta_{2}\left|s_{2}\right|-k_{2} s_{2}^{2}-\eta_{3}\left|s_{3}\right|-k_{3} s_{3}^{2}-\eta_{4}\left|s_{4}\right|-k_{4} s_{4}^{2}
\end{aligned}
$$

If $\eta_{i}>0, k_{i}>0$ and $i=1, \ldots, 4$, for Equation (14), then:

$$
\dot{V}<0
$$

Consequently, according to the Lyapunov second method, a Zhou system with inputs of Equation (11) is globally asymptotically stable, and the proof is complete. 
With initial condition $x(0)=[25,-16,20,-30]$ and $\eta_{i}=\lambda_{i}=0.1, k_{i}=100$ is depicted in Figure 2 . The result in Figure 2 shows that all variables $x_{1}, x_{2}, x_{3}$ and $x_{4}$ of the system were controlled well.

Figure 2. Time responses of the controlled hyperchaotic Zhou system.

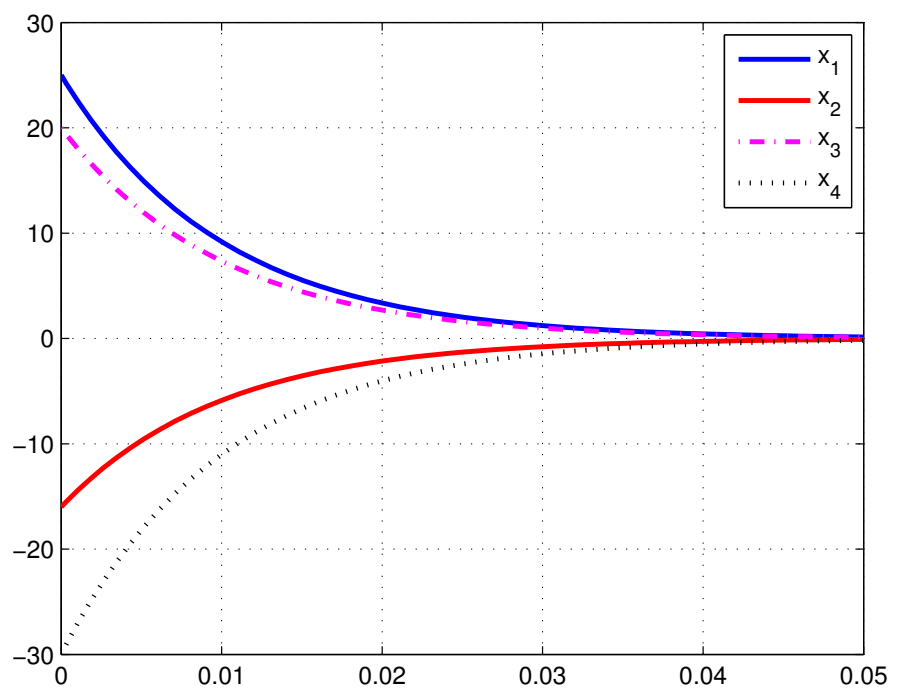

\subsection{Synchronizing of the Hyper-Chaotic Zhou System Using Integral Sliding Mode Control}

In this section, the scheme is further developed based on the result of the previous section. An integral sliding mode controller will be designed for a master-slave hyperchaotic Zhou system. The master and slave systems can be considered as a simple dynamical network with two nodes and one link. In this dynamical network, each node is assumed to have an oscillator, such that its behavior can be described as a hyperchaotic Zhou system. The master hyperchaotic Zhou system is defined as:

$$
\left\{\begin{array}{l}
\dot{x}_{1}=a\left(x_{2}-x_{1}\right)+x_{4} \\
\dot{x}_{2}=c x_{2}-x_{1} x_{3} \\
\dot{x}_{3}=-b x_{3}+x_{1} x_{2} \\
\dot{x}_{4}=d x_{1}+0.5 x_{2} x_{3}
\end{array}\right.
$$

where $a, b, c$ and $d$ are the same parameters as the ones in Equation (2) with initial condition of $x(0)=[25,-16,20,-30]$.

The slave hyperchaotic Zhou system is defined as follows:

$$
\left\{\begin{array}{l}
\dot{y}_{1}=a\left(y_{2}-y_{1}\right)+y_{4}+u_{1} \\
\dot{y}_{2}=c y_{2}-y_{1} y_{3}+u_{2} \\
\dot{y}_{3}=-b y_{3}+y_{1} y_{2}+u_{3} \\
\dot{y}_{4}=d y_{1}+0.5 y_{2} y_{3}+u_{4}
\end{array}\right.
$$

The main objective here is to design a controller to synchronize two of the hyperchaotic systems in Equation (17) with Equation (16), respectively. In the presence of the controller, the slave hyperchaotic system in Equation (17) with arbitrary initial conditions and time intervals by using command signals $u_{i}, i=1, \ldots, 4$, can definitely be synchronized with the master hyperchaotic system in Equation (16). 
The error signal is defined by subtracting Equation (17) from Equation (16):

$$
e_{i}=y_{i}-x_{i}, \quad i=1, \ldots, 4
$$

The derivative of equation in Equation (18) yields:

$$
\dot{e}_{i}=\dot{y}_{i}-\dot{x}_{i}, \quad i=1, \ldots, 4
$$

The sliding surface of the integral sliding mode controller is defined as:

$$
s_{i}=\left(\frac{d}{d t}+\lambda_{i}\right)\left(\int_{0}^{t} e_{i}(\tau) d \tau\right)=e_{i}+\lambda_{i} \int_{0}^{t} e_{i}(\tau) d \tau, \quad i=1, \ldots, 4
$$

The derivative of equation in Equation (20) results:

$$
\dot{s}_{i}=\dot{e}_{i}+\lambda_{i} e_{i}, \quad i=1, \ldots, 4
$$

The Hurwitz condition is realized if $\lambda_{i}>0$ for $i=1, \ldots, 4$.

Equation (21) by considering the exponential reaching law presented by Equation (8) gives:

$$
\left\{\begin{array}{l}
\dot{e}_{1}+\lambda_{1} e_{1}=-\eta_{1} \operatorname{sgn}\left(s_{1}\right)-k_{1} s_{1} \\
\dot{e}_{2}+\lambda_{2} e_{2}=-\eta_{2} \operatorname{sgn}\left(s_{2}\right)-k_{2} s_{2} \\
\dot{e}_{3}+\lambda_{3} e_{3}=-\eta_{3} \operatorname{sgn}\left(s_{3}\right)-k_{3} s_{3} \\
\dot{e}_{4}+\lambda_{4} e_{4}=-\eta_{4} \operatorname{sgn}\left(s_{4}\right)-k_{4} s_{4}
\end{array}\right.
$$

Writing Equation (8) with the provision of Equations (17) and (18):

$$
\left.\begin{array}{rl}
a\left(y_{2}-y_{1}\right)+y_{4}+u_{1}-a\left(x_{2}-x_{1}\right)-x_{4}+\lambda_{1}\left(y_{1}-x_{1}\right) & =-\eta_{1} \operatorname{sgn}\left(s_{1}\right)-k_{1} s_{1} \\
c y_{2}-y_{1} y_{3}+u_{2}-c x_{2}+x_{1} x_{3}+\lambda_{2}\left(y_{2}-x_{2}\right) & =-\eta_{2} \operatorname{sgn}\left(s_{2}\right)-k_{2} s_{2} \\
-b y_{3}+y_{1} y_{2}+u_{3}+b x_{3}-x_{1} x_{2}+\lambda_{3}\left(y_{3}-x_{3}\right) & =-\eta_{3} \operatorname{sgn}\left(s_{3}\right)-k_{3} s_{3} \\
d y_{1}+0.5 y_{2} y_{3}+u_{4}-d x_{1}-0.5 x_{2} x_{3}+\lambda_{4} x_{4} & =-\eta_{4} \operatorname{sgn}\left(s_{4}\right)-k_{4} s_{4}
\end{array}\right\}
$$

Then, the following control laws result:

$$
\left\{\begin{array}{l}
u_{1}=-a\left(e_{2}-e_{1}\right)-e_{4}-\lambda_{1} e_{1}-\eta_{1} \operatorname{sgn}\left(s_{1}\right)-k_{1} s_{1} \\
u_{2}=-c e_{2}+y_{1} y_{3}-x_{1} x_{3}-\lambda_{2} e_{2}-\eta_{2} \operatorname{sgn}\left(s_{2}\right)-k_{2} s_{2} \\
u_{3}=b e_{3}-y_{1} y_{2}-x_{1} x_{2}-\lambda_{3} e_{3}-\eta_{3} \operatorname{sgn}\left(s_{3}\right)-k_{3} s_{3} \\
u_{4}=-d e_{1}-0.5 y_{2} y_{3}+0.5 x_{2} x_{3}-\lambda_{4} e_{4}-\eta_{4} \operatorname{sgn}\left(s_{4}\right)-k_{4} s_{4},
\end{array}\right.
$$

Theorem 2. The response of the hyperchaotic Zhou system in Equation (17) with the arbitrary initial condition $y(0) \in \mathbb{R}^{4}$, using the control laws in Equation (24) and with $\lambda_{i}, \eta_{i}$ and $k_{i}>0$ is same as the response of a system in Equation (16). This means Equation (18) is globally asymptotically stable.

Proof. This is the same as the proof of Theorem 1 that is presented from Equation (12) to Equation (15).

With initial conditions $x(0)=[25,-16,20,-30]$, by choosing controller parameters as $\eta_{i}=\lambda_{i}=0.1$ and $k_{i}=100$ for $i=1, \ldots, 4$, the state variables trajectories are shown in Figure 3 . In this figure, the controller is off for $t<2$ and is on for $t \geq 2$. Simulation results in Figure 3 show that all four slave states 
are converging to the master ones, and also, Figure 4 shows that the error of the controller is converging to zero, which means that the synchronization is achieved pretty well using the proposed controller.

Figure 3. Trajectories of the synchronization states $x_{1}(\mathbf{a}), x_{2}(\mathbf{b}), x_{3}(\mathbf{c})$ and $x_{4}(\mathbf{d})$ (the controller is off for $t<2$ and is on for $t \geq 2$ ).

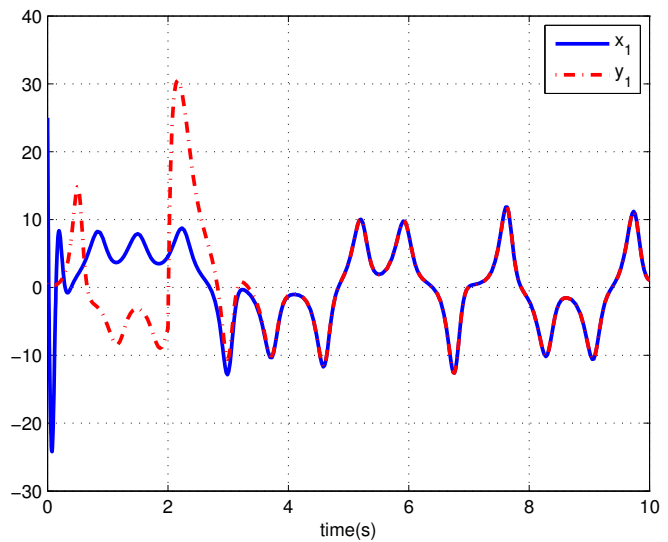

(a)

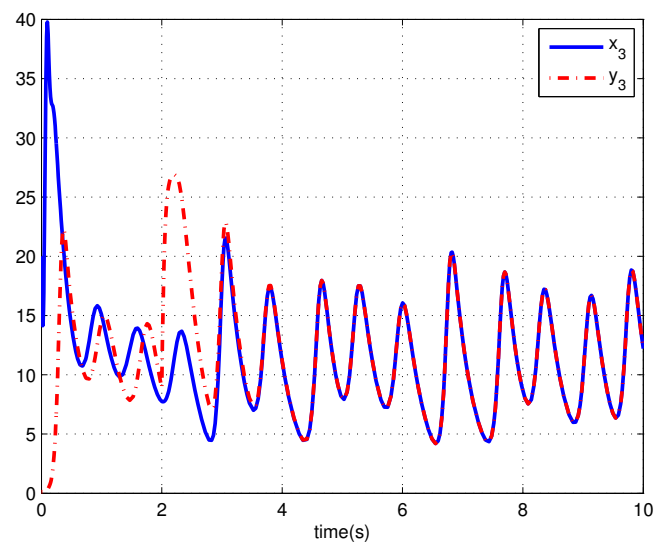

(c)

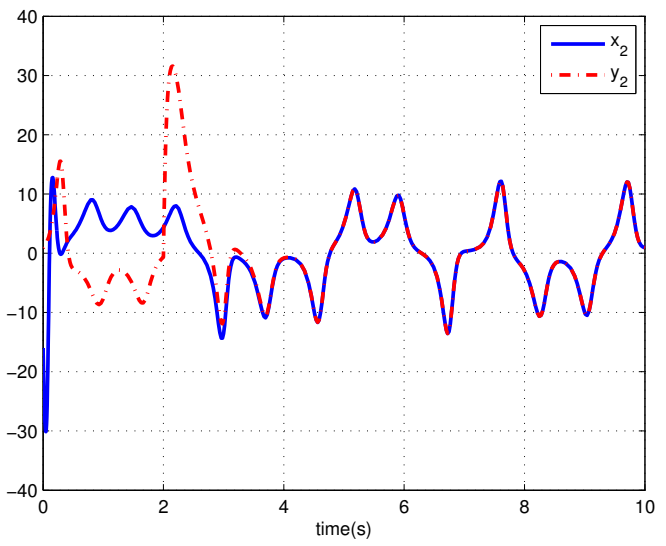

(b)

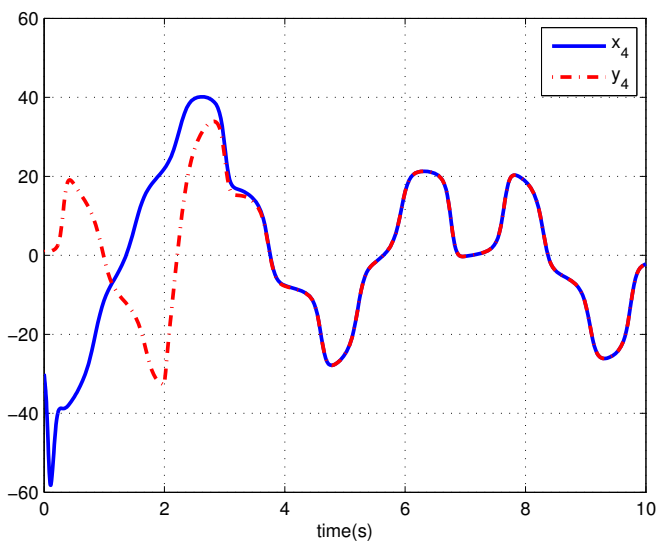

(d)

Figure 4. Trajectories of the synchronization errors of $e_{1}, e_{2}, e_{3}$ and $e_{4}$ (the controller is off for $t<2$ and is on for $t \geq 2$ ).

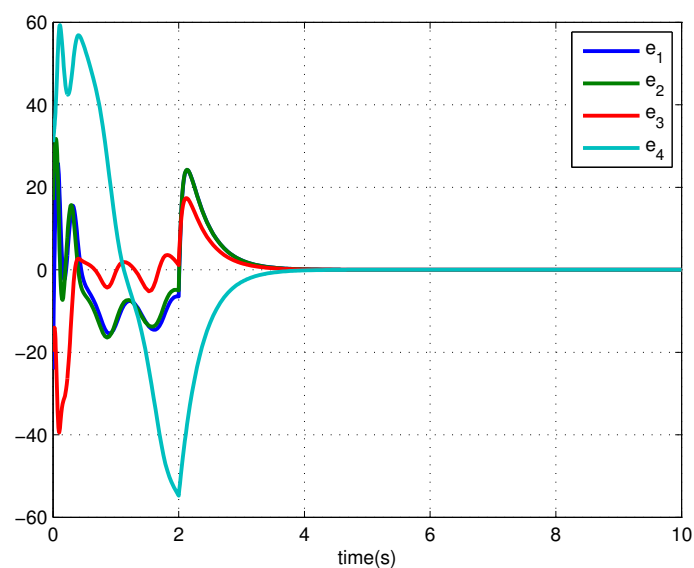


3.2. Parameter Estimation and Synchronization of the Hyperchaotic Zhou System Using an Adaptive Integral Sliding Mode Control

In this section, an adaptive integral sliding mode controller is proposed to synchronize two slave and master hyperchaotic system.

Suppose that the following master hyperchaotic Zhou system contains the unknown parameters:

$$
\left\{\begin{array}{l}
\dot{x}_{1}=a\left(x_{2}-x_{1}\right)+x_{4} \\
\dot{x}_{2}=c x_{2}-x_{1} x_{3} \\
\dot{x}_{3}=-b x_{3}+x_{1} x_{2} \\
\dot{x}_{4}=d x_{1}+0.5 x_{2} x_{3}
\end{array}\right.
$$

where $a, b, c, d$ are the same constant parameters, but unknown.

The system in Equation (25) with $a=35, b=3, c=12$ and $d=1$ will show a hyperchaotic behavior.

The following system is considered as a slave system:

$$
\left\{\begin{array}{l}
\dot{y}_{1}=a(t)\left(y_{2}-y_{1}\right)+y_{4}+u_{1} \\
\dot{y}_{2}=c(t) y_{2}-y_{1} y_{3}+u_{2} \\
\dot{y}_{3}=-b(t) y_{3}+y_{1} y_{2}+u_{3} \\
\dot{y}_{4}=d(t) y_{1}+0.5 y_{2} y_{3}+u_{4}
\end{array}\right.
$$

where $u_{i}, i=1, \ldots, 4$, are nonlinear time variant command signals, which are used to synchronize the two systems in Equation (26), respectively. Furthermore, $a(t), b(t), c(t)$ and $d(t)$ are instant estimations of $a, b, c$ and $d$ in time $t$. For the estimation of unknown parameters of the slave system in Equation (26), an adaptive law will be calculated and used in the synchronizing process.

Through subtraction of Equation (26) from Equation (25) and considering the definition of error signal and its derivative, the resulting equations are as follows:

$$
\left\{\begin{array}{l}
\dot{e}_{1}=a(t)\left(y_{2}-y_{1}\right)+y_{4}+u_{1}-a\left(x_{2}-x_{1}\right)-x_{4} \\
\dot{e}_{2}=c(t) y_{2}-y_{1} y_{3}+u_{2}-c x_{2}+x_{1} x_{3} \\
\dot{e}_{3}=-b(t) y_{3}+y_{1} y_{2}+u_{3}+b x_{3}-x_{1} x_{2} \\
\dot{e}_{4}=d(t) y_{1}+0.5 y_{2} y_{3}+u_{4}-d x_{1}-0.5 x_{2} x_{3}
\end{array}\right.
$$

Let us consider the following Lyapunov function:

$$
V\left(s_{1}, s_{2}, s_{3}, s_{4}, \tilde{a}, \tilde{b}, \tilde{c}, \tilde{d}\right)=\frac{1}{2}\left(s_{1}^{2}+s_{2}^{2}+s_{3}^{2}+s_{4}^{2}+\tilde{a}^{2}+\tilde{b}^{2}+\tilde{c}^{2}+\tilde{d}^{2}\right)
$$

where $\tilde{a}, \tilde{b}, \tilde{c}$ and $\tilde{d}$ are defined as below:

$$
\left\{\begin{array}{l}
\tilde{a}=a(t)-a \\
\tilde{b}=b(t)-b \\
\tilde{c}=c(t)-c \\
\tilde{d}=d(t)-d
\end{array}\right.
$$

The derivative of the function in Equation (29) yields:

$$
\begin{aligned}
\frac{d V}{d t}= & s_{1} \dot{s}_{1}+s_{2} \dot{s}_{2}+s_{3} \dot{s}_{3}+s_{4} \dot{s}_{4}+\dot{a}(t) \tilde{a}+\dot{b}(t) \tilde{b}+\dot{c}(t) \tilde{c}+\dot{d}(t) \tilde{d} \\
= & s_{1}\left(\dot{e}_{1}+\lambda_{1} e_{1}\right)+s_{2}\left(\dot{e}_{2}+\lambda_{2} e_{2}\right)+s_{3}\left(\dot{e}_{3}+\lambda_{3} e_{3}\right)+s_{4}\left(\dot{e}_{4}+\lambda_{4} e_{4}\right) \\
& +\dot{a}(t) \tilde{a}+\dot{b}(t) \tilde{b}+\dot{c}(t) \tilde{c}+\dot{d}(t) \tilde{d}
\end{aligned}
$$


Considering Equation (27), Equation (30) can be written as:

$$
\begin{aligned}
\frac{d V}{d t}= & s_{1}\left(a(t)\left(y_{2}-y_{1}\right)+y_{4}+u_{1}-a\left(x_{2}-x_{1}\right)-x_{4}+\lambda_{1} e_{1}+a(t)\left(x_{2}-x_{1}\right)-a(t)\left(x_{2}-x_{1}\right)\right) \\
& +s_{2}\left(c(t) y_{2}-y_{1} y_{3}+u_{2}-c x_{2}+x_{1} x_{3}+\lambda_{2} e_{2}+c(t) x_{2}-c(t) x_{2}\right) \\
& +s_{3}\left(-b(t) y_{3}+y_{1} y_{2}+u_{3}+b x_{3}-x_{1} x_{2}+\lambda_{3} e_{3}+b(t) x_{3}-b(t) x_{3}\right) \\
& +s_{4}\left(d(t) y_{1}+0.5 y_{2} y_{3}+u 4-d x_{1}-0.5 x_{2} x_{3}+\lambda_{4} e_{4}+d(t) x_{1}-d(t) x_{1}\right) \\
& +\dot{a}(t) \tilde{a}+\dot{b}(t) \tilde{b}+\dot{c}(t) \tilde{c}+\dot{c}(t) \tilde{c}+\dot{c}(t) \tilde{c}+\dot{d}(t) \tilde{d} \\
= & s_{1}\left(a(t)\left(e_{2}-e_{1}\right)+e_{4}+u_{1}+\lambda_{1} e_{1}\right) \\
& +s_{2}\left(c(t) e_{2}-y_{1} y_{3}+u_{2}+x_{1} x_{3}+\lambda_{2} e_{2}\right) \\
& +s_{3}\left(-b(t) e_{3}+y_{1} y_{2}+u_{3}-x_{1} x_{2}+\lambda_{3} e_{3}\right) \\
& +s_{4}\left(d(t) e_{1}+0.5 y_{2} y_{3}+u_{4}-0.5 x_{2} x_{3}+\lambda_{4} e_{4}\right) \\
= & s_{1}(a(t)-a)\left(x_{2}-x_{1}\right)+\dot{a}(t) \tilde{a}+\dot{b}(t) \tilde{b}-s_{3}(b(t)-b) x_{3}+\dot{c}(t) \tilde{c} \\
& +s_{2}(c(t)-c) x_{2}+\dot{d}(t) \tilde{d}+s_{4}(d(t)-d) x_{1} \\
= & s_{1}\left(a(t)\left(e_{2}-e_{1}\right)+e_{4}+u_{1}+\lambda_{1} e_{1}\right) \\
& +s_{2}\left(c(t) e_{2}-y_{1} y_{3}+u_{2}+x_{1} x_{3}+\lambda_{2} e_{2}\right) \\
& +s_{3}\left(-b(t) e_{3}+y_{1} y_{2}+u_{3}-x_{1} x_{2}+\lambda_{3} e_{3}\right) \\
& +s_{4}\left(d(t) e_{1}+0.5 y_{2} y_{3}+u_{4}-0.5 x_{2} x_{3}+\lambda_{4} e_{4}\right) \\
& +\left(\dot{a}(t)+s_{1}\left(x_{2}-x_{1}\right) \tilde{a}\right)+\left(\dot{b}(t)-s_{3} x_{3}\right) \tilde{b}+\left(\dot{c}(t)+s_{2} x_{2}\right) \tilde{c}+\left(\dot{d}(t)+s_{4} x_{1}\right) \tilde{d} \\
&
\end{aligned}
$$

Based on the following control laws:

$$
\left\{\begin{array}{l}
u_{1}=-a(t)\left(e_{2}-e_{1}\right)-e_{4}-\lambda_{1} e_{1}-\eta_{1} \operatorname{sgn}\left(s_{1}\right)-k_{1} s_{1} \\
u_{2}=-c(t) e_{2}+y_{1} y_{2}-x_{1} x_{3}-\lambda_{2} e_{2}-\eta_{2} \operatorname{sgn}\left(s_{2}\right)-k_{2} s_{2} \\
u_{3}=b(t) e_{3}-y_{1} y_{2}+x_{1} x_{2}-\lambda_{3} e_{3}-\eta_{3} \operatorname{sgn}\left(s_{3}\right)-k_{3} s_{3} \\
u_{4}=-d(t) e_{1}-0.5 y_{2} y_{3}+0.5 x_{2} x_{3}-\lambda_{4} e_{4}-\eta_{4} \operatorname{sgn}\left(s_{4}\right)-k_{4} s_{4},
\end{array}\right.
$$

and the following adaptive laws:

$$
\left\{\begin{array}{l}
\dot{a}(t)=-s_{1}\left(x_{2}-x_{1}\right) \\
\dot{b}(t)=s_{3} x_{3} \\
\dot{c}(t)=-s_{2} x_{2} \\
\dot{d}(t)=-s_{4} x_{1}
\end{array}\right.
$$

Equation (31) can be rewritten as below with the conditions of Equations (32) and (33),

$$
\frac{d V}{d t}=-\eta_{1}\left|s_{1}\right|-k_{1} s_{1}^{2}-\eta_{2}\left|s_{2}\right|-k_{2} s_{2}^{2}-\eta_{3}\left|s_{3}\right|-k_{3} s_{3}^{2}-\eta_{4}\left|s_{4}\right|-k_{4} s_{4}^{2}
$$

Choosing $\eta_{i}>0, k_{i}>0, i=1, \ldots, 4$ for Equation (34) will result in:

$$
\frac{d V}{d t}<0
$$

This yields the following theorem: 
Theorem 3. The response of the slave hyperchaotic Zhou system in Equation (26) with the arbitrary initial condition $y(0) \in \mathbb{R}^{4}$, using the control laws in Equation (32) with $\lambda_{i}, \eta_{i}, k_{i}>0$ and the adaption laws in Equation (33), tends toward the response of the master system in Equation (25) with unknown parameters. This means that the error signals in Equation (27) and the parameter errors in Equation (29) are globally asymptotically stable and converge to zero.

\section{Results and Discussion}

In this section, the performance of the proposed scheme on the Zhou system is analyzed. Results of an enhanced method using Vaidyanathan's scheme [26] is also presented. Synchronization and estimation of unknown parameters on both the hyperchaotic Zhou system and the master-slave system is investigated.

The parameter values as in the hyperchaotic case are assumed as $a=35, b=3, c=12$ and $d=1$.

Comparing the proposed scheme and the Vaidyanathan's scheme [26], it is found that both schemes are implemented with a same initial condition, where the master system uses $x(0)=\left[\begin{array}{ll}25-16 & 20-30\end{array}\right]$ and the initial conditions of the slave system for two methods are defined as $y(0)=\left[\begin{array}{llll}14 & 28 & -10 & 6\end{array}\right]$. Constant control parameters for this simulation are valued by $k_{i}=20, \eta_{i}=0.1$ and $\lambda_{i}=100$, where $i=1, \cdots, 4$.

Figure 5. Comparison between simulations results of the proposed method and the previously known method: $x_{1}(\mathbf{a}), x_{2}(\mathbf{b}), x_{3}(\mathbf{c})$ and $x_{4}(\mathbf{d})$.

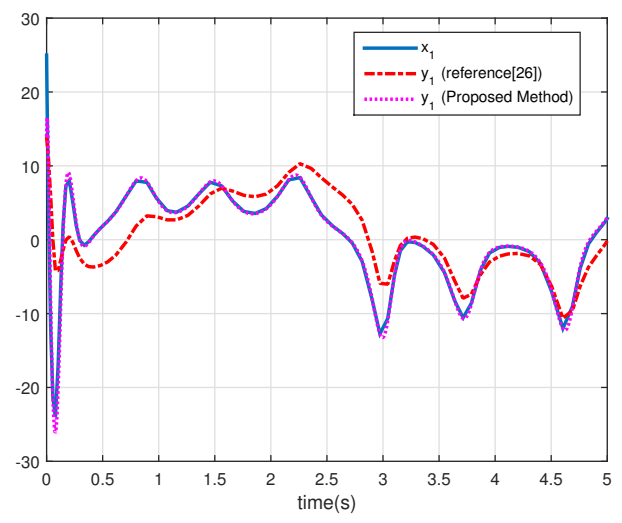

(a)

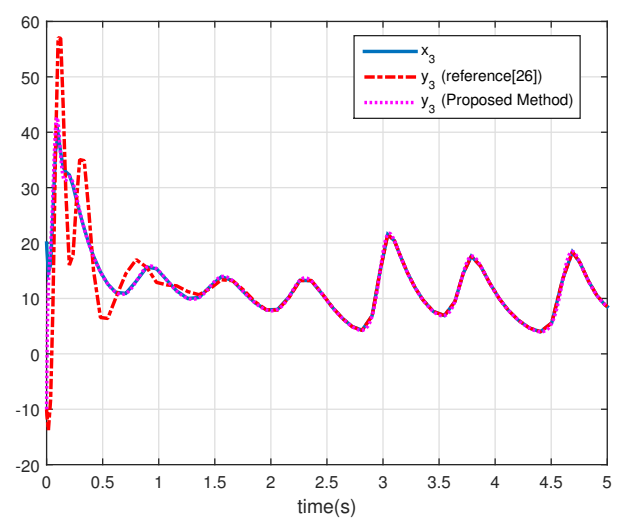

(c)

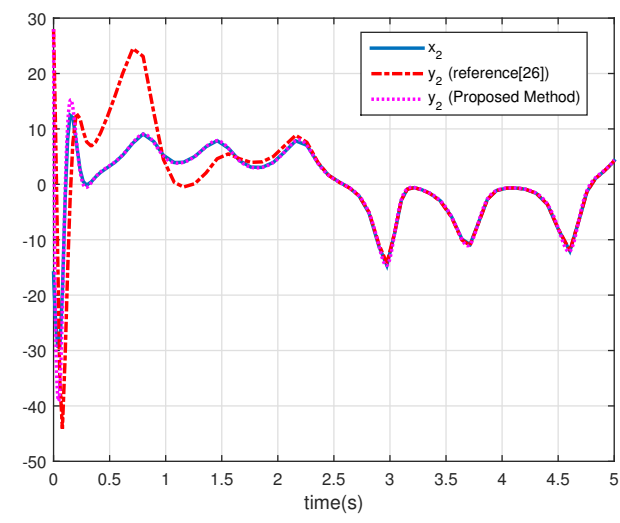

(b)

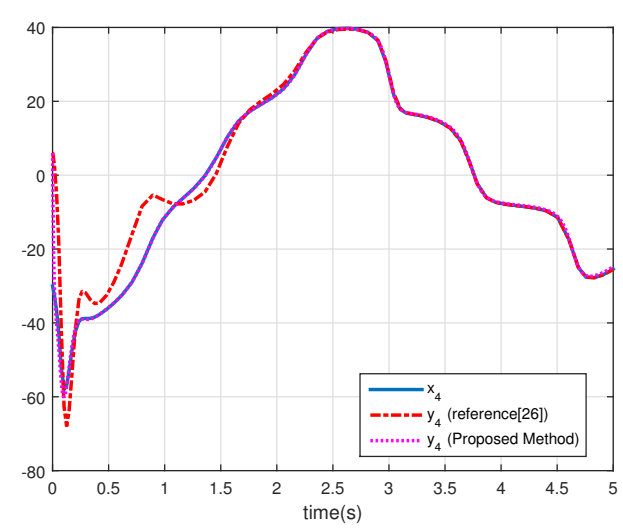

(d) 
Furthermore, $k_{i}=5, \eta_{i}=0.1$ are considered as the control parameters in Vaidyanathan's scheme [26]. With simulation duration $\mathrm{T}=5 \mathrm{~s}$, the results are shown in Figures 5 and 6. Compared with Vaidyanathan's scheme [26], the proposed method shows a faster response in the synchronization of the slave system parameters with the ones in the master system. With the proposed method, after passing time of $\mathrm{T}=5 \mathrm{~s}$, the $x_{1}$ was synchronized with the master system, while with the method that was proposed in [26], the $x_{1}$ could not be synchronized yet with related referenced parameters in the master system.

Figure 6. Comparison between the simulation errors of the proposed method and the previously known method $e_{1}, e_{2}, e_{3}$ and $e_{4}$.

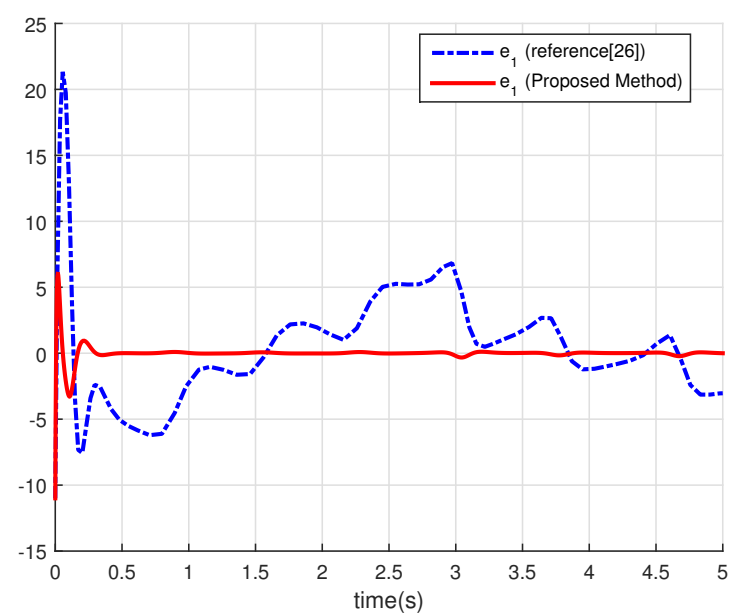

(a)

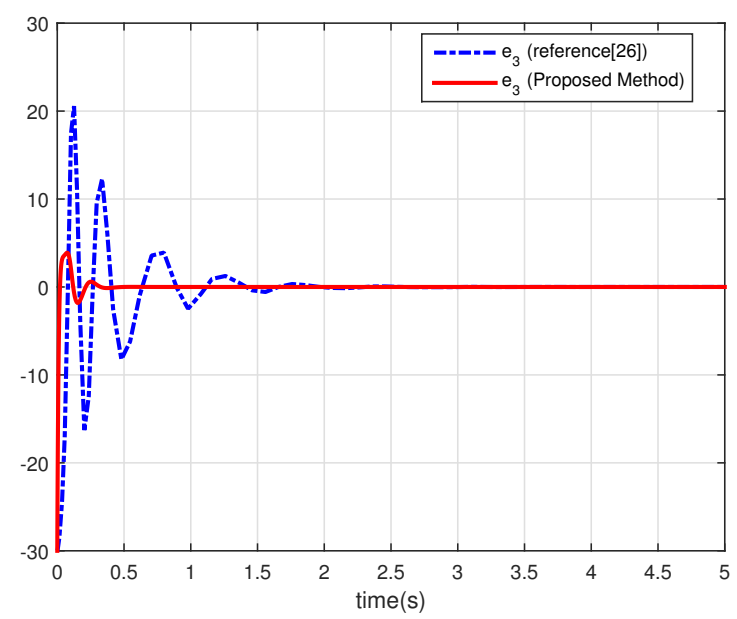

(c)

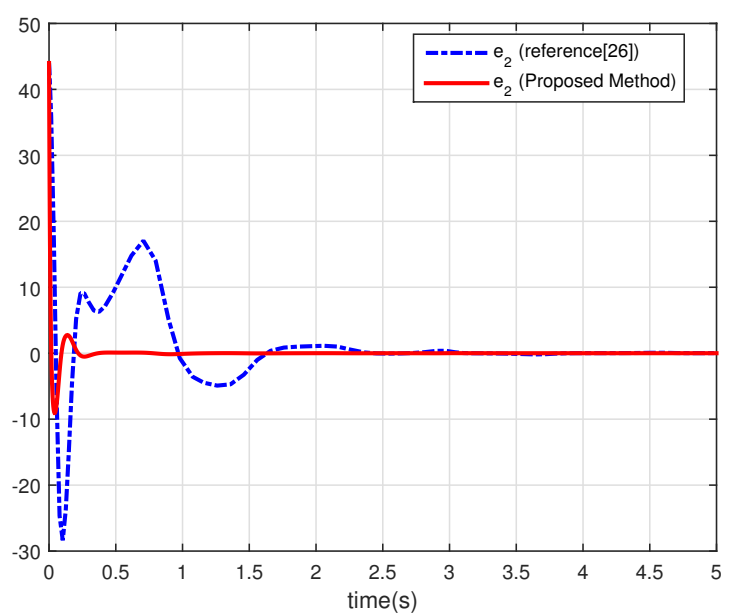

(b)

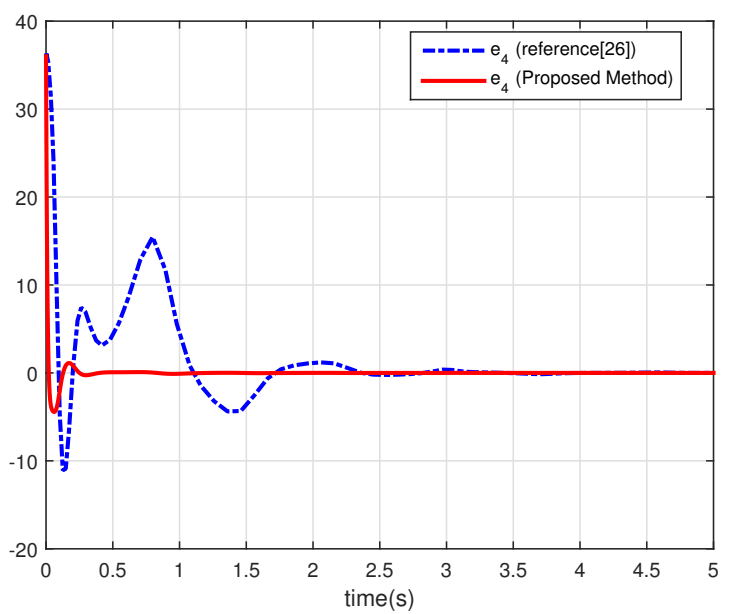

(d)

Adaptation performance of the slave parameters and convergence of the slave system parameters toward the ones in the master system are presented in Figure 7.

Based on Figure 7, the parameter of $a(t)$ in both schemes shows a slow increasing rate in attempting to be close to the related referenced parameter in the master system, which means $a=35$. However, the results show that with the performance of the proposed method, other initial unknown parameters in the slave system could converge to known parameters in the master system with faster responses and less 
oscillations, when compared with the performance of the method in [26]. As has been shown in both the synchronization process and the identification of unknown parameters for the slave system, the proposed scheme can have more efficient performance compared with other methods, such as the method in [26].

Figure 7. Comparison results of parameter convergence between two schemes: time history of parameters $a, b, c$ and $d$ in the proposed scheme (a) and Vaidyanathan's scheme (b).

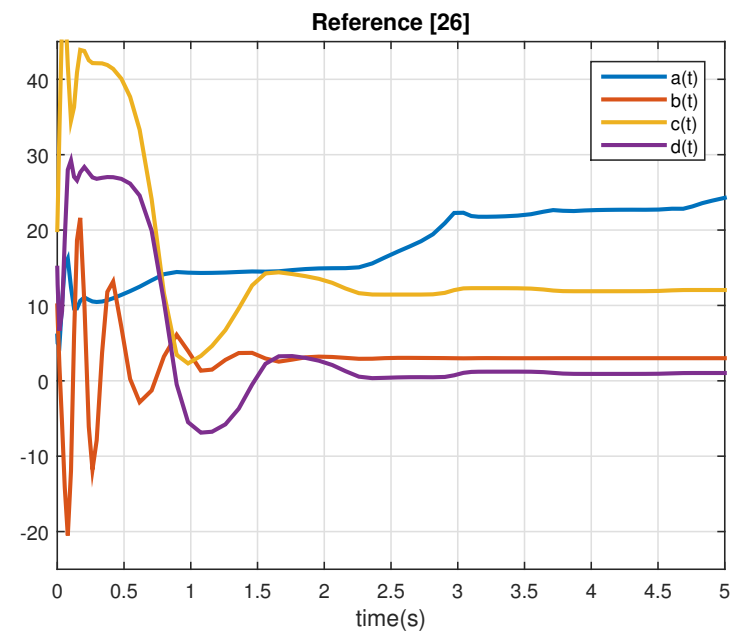

(a)

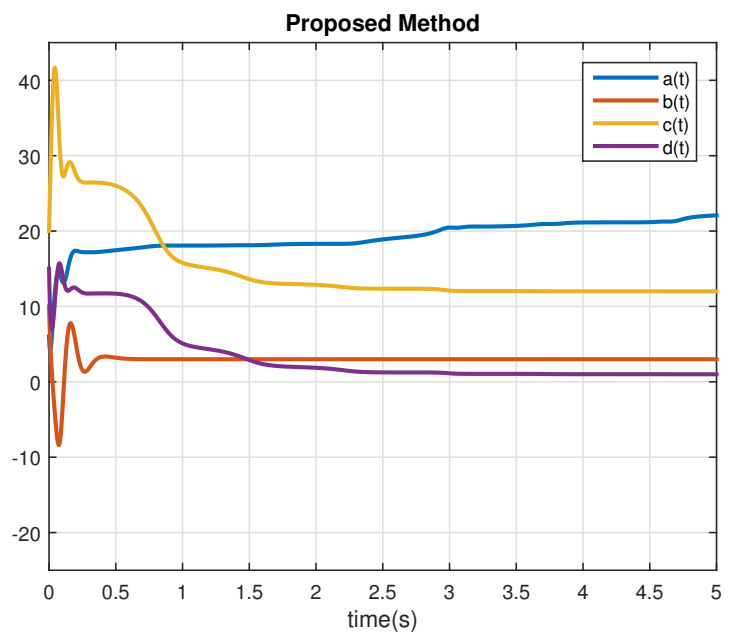

(b)

\section{Conclusions}

An adaptive integral sliding mode controller is proposed to control and synchronize the hyperchaotic Zhou system. First, a sliding mode controller is designed to stabilize the hyperchaotic Zhou system to its unstable equilibrium at the origin. Then, an adaptive procedure is introduced to improve the basic controller to an adaptive integral sliding mode controller. Using the proposed adaptive controller and extracted command laws, both slave and master hyperchaotic Zhou systems converge to the same responses. Stability analysis for the developed controller in each stage in the scheme is proven by use of the Lyapunov theory. The results with the proposed control scheme show that the adaptive integral sliding mode controller can control the hyperchaotic system efficiently, even when the Zhou system modeling has uncertainty due to its unknown parameters. For future work, the proposed scheme can be applied to a complex network with more nodes for an efficient chaos synchronization.

\section{Author Contributions}

Both authors contributed to the initial motivation of the work, to the research and numerical experiments and to the writing. Both authors have read and approved the final manuscript.

\section{Conflicts of Interest}

The authors declare no conflict of interest. 


\section{References}

1. Rössler, O. An Equation for Hyperchaos. Phys. Lett. A 1979, 57, 155-157.

2. Ning, C.Z.; Haken, H. Detuned lasers and the Complex Lorenz equations: Subcritical and supercritical Hopf bifurcations. Phys. Rev. A 1990, 41, 3826-3837.

3. Kapitaniak, T.; Chua, L. Hyperchaotic Attractors of Undirectionally-Coupled Chua's Circuits. Int. J. Bifurc. Chaos 2006, 4, 477-482.

4. Li, Y.; Tang, W.; Chen, G. Generating hyperchaos via state feedback control. Int. J. Bifurc. Chaos 2005, 15, 3367-3375.

5. Chen, A.; Lu, J.; Lü, J.; Yu, S. Generating hyperchaotic Lü attractor via state feedback control. Physica A 2006, 364, 103-110.

6. Cai, G.; Huang, J.; Tian, L.; Wang, Q. Adaptive control and slow manifold analysis of a new chaotic system. Int. J. Nonlinear Sci. 2006, 2, 50-60.

7. Sun, M.; Tian, L.; Jiang, S.; Xu, J. Feedback control and adaptive control of the energy resource chaotic system. Chaos Solitons Fractals 2007, 32, 1725-1734.

8. Ott, E.; Grebogi, C.; Yorke, J.A. Controlling chaos. Phys. Rev. Lett. 1990, 64, 1196-1199.

9. Ge, S.S.; Wang, C.; Lee, T.H. Adaptive backstepping control of a class of chaotic systems. Int. J. Bifurc. Chaos 2000, 10, 1149-1156.

10. Pecora, L.M.; Carroll, T. Synchronization in chaotic systems. Phys. Rev. Lett. 1990, 64, 821-824.

11. Lakshmanan, M.; Murali, K. Chaos in Nonlinear Oscillators: Controlling and Synchronization, Volume 13; World Scientific: Singapore, Singapore, 1996.

12. Han, S.K.; Kurrer, C.; Kuramoto, Y. Dephasing and bursting in coupled neural oscillators. Phys. Rev. Lett. 1995, 75, 3190-3193.

13. Blasius, B.; Huppert, A.; Stone, L. Complex dynamics and phase synchronization in spatially extended ecological systems. Nature 1999, 399, 354-359.

14. Feki, M. An adaptive chaos synchronization scheme applied to secure communication. Chaos Solitons Fractals 2003, 18, 141-148.

15. Murali, K.; Lakshmanan, M. Secure communication using a compound signal from generalized synchronizable chaotic systems. Phys. Lett. A 1998, 241, 303-310.

16. Garfinkel, A. Controlling cardiac chaos. Science 1992, 257, 1230-1235.

17. Nakamura, Y.; Sekiguchi, A. The chaotic mobile robot. IEEE Trans. Robot. Autom. 2001, 17, 898-904.

18. Wang, X. Complex networks: Topology, dynamics and synchronization. Int. J. Bifurc. Chaos 2002, 12, 885-916.

19. Ho, M. Synchronization of two different systems by using generalized active control. Phys. Lett. A 2002, 301, 424-428.

20. Tang, R.A.; Liu, Y.L.; Xue, J.K. An extended active control for chaos synchronization. Phys. Lett. A 2009, 373, 1449-1454.

21. Sundarapandian, V. Global chaos synchronization of Lorenz and Pehlivan chaotic systems by nonlinear control. Int. J. Adv. Sci. Tech. 2011, 2, 19-28. 
22. Vaidyanathan, S.; Rajagopal, K. Global chaos synchronization of hyperchaotic Pang and hyperchaotic Wang systems via adaptive control. Int. J. Soft Comput. 2012, 7, 28-37.

23. Liao, T.L.; Tsai, S. Adaptive synchronization of chaotic systems and its application to secure communications. Chaos Solitons Fractals 2000, 11, 1387-1396.

24. Park, J.; Kwon, O. A novel criterion for delayed feedback control of time-delay chaotic systems. Chaos Solitons Fractals 2005, 23, 495-501.

25. Zhao, J.; Lu, J. Using sampled-data feedback control and linear feedback synchronization in a new hyperchaotic system. Chaos Solitons Fractals 2008, 35, 376-382.

26. Vaidyanathan, S. Adaptive controller and synchronizer design for hyperchaotic Zhou system with unknown parameters. Int. J. Inf. Tech. Model. Comput. 2013, 1, 18-32.

27. Konishi, K.; Hirai, M.; Kokame, H. Sliding mode control for a class of chaotic systems. Phys. Lett. A 1998, 245, 511-517.

28. Hou, Y.; Liau, B.; Chen, H. Synchronization of unified chaotic systems using sliding mode controller. Math. Prob. Eng. 2012, 2012, doi:10.1155/2012/632712.

29. Vaidyanathan, S.; Rasappan, S. Global chaos synchronization for Windmi and Coullet chaotic systems using active control. J. Control Eng. Tech. 2013, 3, 69-78.

30. Wu, J.; Zhang, J.; Liu, Z. Neural network sliding mode control based on improved particle swarm optimization algorithm for discrete-time chaotic systems. Control Decis. 2013, 28, 1094-1098.

31. Yang, C.; Ou, C. Adaptive terminal sliding mode control subject to input nonlinearity for synchronization of chaotic gyros. Commun. Nonlinear Sci. Numer. Simul. 2013, 18, 682-691.

32. Pai, M.C. Global synchronization of uncertain chaotic systems via discrete-time sliding mode control. Appl. Math. Comput. 2014, 227, 663-671.

33. Zhang, X.; Liu, X.; Zhu, Q. Adaptive chatter free sliding mode control for a class of uncertain chaotic systems. Appl. Math. Comput. 2014, 232, 431-435.

34. Uyaroğlu, Y.K; Kocamaz, U.E.; Göksu, A.; Can, E. Sliding mode control of hyperchaotic finance system. In Proceedings of International Work-Conference on Time Series (ITISE 2014), Granada, Spain, 25-27 June 2014; pp. 260-268.

35. Hahn, W.; Baartz, A. Stability of Motion; Springer: Berlin, Germany, 1967.

36. Utkin, V.I. Sliding mode control design principles and applications to electric drives. IEEE Trans. Ind. Electron. 1993, 40, 23-36.

37. Zhou, P.; Cao, Y.; Cheng, X. A new hyperchaos system and its circuit simulation by EWB. Chin. Phys. B 2009, 18, 1394-1398.

38. Slotine, J.; Li, W. Applied Nonlinear Control; Prentice-Hall: Englewood Cliffs, NJ, USA, 1991.

(C) 2014 by the authors; licensee MDPI, Basel, Switzerland. This article is an open access article distributed under the terms and conditions of the Creative Commons Attribution license (http://creativecommons.org/licenses/by/4.0/). 